http://doi.org/10.15359/ree.14-Ext.12

\title{
Dimes y diretes de un proyecto de centro: el caso de la Escuela Laboratorio en Heredia
}

\section{The Pros and Cons of a Field Project: The case of the Laboratory School in Heredia}

\author{
Cecilia Dobles Trejos ${ }^{l}$ \\ División de Educación Básica del \\ Centro de Investigación y Docencia en Educación (CIDE) de la \\ Universidad Nacional \\ Heredia, Costa Rica \\ Dora María Hernández Vargas ${ }^{2}$ \\ División de Educación Básica del \\ Centro de Investigación y Docencia en Educación (CIDE) de la \\ Universidad Nacional \\ Heredia, Costa Rica \\ Heidy León Arce \\ División de Educación Básica del \\ Centro de Investigación y Docencia en Educación (CIDE) de la \\ Universidad Nacional \\ Heredia, Costa Rica
}

Recibido 03 de febrero de 2010 • Aceptado 17 de marzo de 2010

\footnotetext{
Antropóloga social. Profesora e investigadora de la División de Educación Básica del Centro de Investigación y Docencia en Educación (CIDE) de la Universidad Nacional, Costa Rica. Además ha sido profesora de la Escuela de Antropología y Sociología de la Universidad de Costa Rica. Se desempeña también como consultora independiente realizando investigaciones y ejecutando proyectos en los campos de la gestión cultural, derechos de poblaciones específicas y gestión de las organizaciones de la sociedad civil.

Correo Electrónico: ceciliadobles@ice.co.cr

2 Académica de la División de Educación Básica, del Centro de Investigación y Docencia en Educación (CIDE) de la Universidad Nacional, Costa Rica. Master en Pedagogía con énfasis en Diversidad en los Procesos Educativos, por la Universidad Nacional, Costa Rica, y licenciada en Ciencias de la Educación con énfasis en Educación Preescolar por la misma Universidad. Egresada del Conservatorio Castella, Costa Rica, con énfasis en Canto y Creación Literaria, continuó sus estudios de canto en la Escuela de Artes Musicales de la Universidad de Costa Rica. Autora de artículos publicados en la Revista EDUCARE sobre el tema de la diversidad. Correo electrónico: dorahv@gmail.com

3 Licenciada en Educación Especial con énfasis en Integración. Bachiller en Educación Especial. Diplomado en Ciencias de la Educación con concentración en Educación Básica I y II ciclos de la Universidad Nacional, Costa Rica. Tiene experiencia docente en instituciones costarricenses, en las que ha trabajado en educación primaria y en servicios de Educación Especial. Actualmente es académica de la División de Educación Básica del Centro de Investigación y Docencia en Educación (CIDE) de la Universidad Nacional, Costa Rica. Labora para el Ministerio de Educación Pública, Costa Rica, en el área de Retardo Mental en el servicio de Estimulación Temprana. Cursa la Maestría en Pedagogía con Énfasis en Diversidad de los Procesos Educativos. Correo electrónico: heidyleon15@hotmail.com
} 
Resumen. En este artículo se hace una revisión de algunas de las características más relevantes de la Escuela Laboratorio de Heredia, Costa Rica, que permiten reconocer los aciertos y tropiezos que una experiencia innovadora de un proyecto de centro, logra y enfrenta dentro del sistema educativo nacional. Se hace una descripción del modelo pedagógico de la escuela y se incluye, además, la visión de los distintos involucrados en cuanto a esta propuesta. Se evidencian las distintas posiciones de los actores que viven y reflexionan cotidianamente en el centro educativo.

Palabras clave. Modelo pedagógico, identidad escolar, proyecto de escuela, proyecto de centro

\begin{abstract}
This article reviews some of the most important characteristics of the Laboratory School in Heredia, Costa Rica, for recognizing the success aspects and setbacks that an innovative experience of an educational project has within the national education system. It is a description of the pedagogical model of the school and includes the vision that different participants have in this educational project, around which it is showed the different positions of the actors who live and reflect daily in the school.
\end{abstract}

Key words. Pedagogical model, school identity, school project, school-based management.

\title{
Introducción
}

En este escrito se lleva a cabo una revisión de las características más relevantes del centro educativo Escuela Laboratorio de Heredia, las cuales permiten reconocer los aciertos y los tropiezos que debe enfrentar una experiencia innovadora de un proyecto de centro, dentro del sistema educativo nacional.

El contenido de este documento se basa en los resultados de una investigación, titulada "El reconocimiento de las identidades educativas en la construcción de un proyecto de escuela", realizada durante los años 2006 y 2007.

Las investigadoras consideraron importante hacer un breve recorrido por las condiciones del contexto educativo, en general, y por la historia del centro de educativo, en particular, con el fin de tener elementos para entender la discusión y los conflictos generados a raíz de la puesta en práctica del nuevo modelo pedagógico de la institución.

Por lo anterior, se hace una descripción del modelo pedagógico y se incluye, además, la visión que tienen los distintos involucrados sobre esta propuesta, en torno a la cual se evidencian las distintas posiciones de los actores que viven y reflexionan cotidianamente en este centro.

\section{Contexto de la Escuela Laboratorio en Heredia}

La región educativa está organizada, administrativamente, en ocho circuitos. Los circuitos 01 y 02 abarcan tanto distritos centrales como urbanos de esta provincia. Los centros educativos de los circuitos 03 y 05 están localizados en áreas más urbanas (60\%) que rurales (40\%); inversamente a lo que sucede en el 04, en el que el paisaje es más rural (60\%) que urbano (40\%). Los circuitos escolares 06,07 y 08 son completamente rurales; en ellos, el turismo y la actividad agro exportadora de banano, piña y tubérculos son las principales fuentes de trabajo.

La Escuela Laboratorio está en el Circuito 04 de la Dirección Regional de Educación de Heredia. Ese circuito comprende 33 escuelas ubicadas en los cantones de San Rafael y Barva. 
Tabla 1

Radio de acción geográfica del Circuito 04 de la Región Educativa de Heredia

\begin{tabular}{|c|l|l|}
\hline Circuito & Cantones & Distritos \\
\hline & Barva & San José de la Montaña \\
& & $\begin{array}{l}\text { San Pedro, San Pablo } \\
\text { Santa Lucía, Barva } \\
\text { San Roque }\end{array}$ \\
& San Rafael & $\begin{array}{l}\text { San Rafael, Concepción } \\
\text { Los Ángeles, San Josecito }\end{array}$ \\
\hline
\end{tabular}

Nota. Departamento de Estadísticas del MEP, 2006.

Dentro del sistema regular, la Región Educativa de Heredia cuenta con una población aproximada de 75,205 estudiantes del sistema de educación tradicional y no tradicional (las tele secundarias).

Tabla 2

Matrícula total (estimada) I y II Ciclos. Según circuito y centros públicos y privados, 2006

\begin{tabular}{|c|c|c|}
\hline \multicolumn{3}{|c|}{ I y II ciclos } \\
\hline & Públicos & Privados \\
\hline $\mathrm{C} 1\left(^{*}\right)$ & 4108 & 198 \\
\hline $\mathrm{C} 2$ & 8157 & 681 \\
\hline $\mathrm{C} 3$ & 7898 & 1118 \\
\hline $\mathrm{C} 4$ & 7058 & 722 \\
\hline C5 & 6261 & 1278 \\
\hline C6 & 2680 & - \\
\hline C7 & 3134 & - \\
\hline C8 & 2703 & 3997 \\
\hline Total & 41999 & \\
\hline Total & & \\
\hline
\end{tabular}

Nota. Tomado del Departamento de Estadísticas del MEP, 2006.

* La C mayúscula hace referencia al Circuito escolar

El $61 \%$ de la población estimada está cursando I y II ciclos, y un $91 \%$ lo hace dentro del sistema de educación pública. Según datos del Ministerio de Planificación Nacional y Política Económica (2005), para el año 2004, la tasa neta regional de cobertura en educación primaria era del 93,5 o sea, un poco inferior al promedio nacional (99,5\%). Asimismo, la tasa de deserción en primaria es de $2,8 \%$, ligeramente inferior al promedio nacional $(3,3 \%)$, igual que el porcentaje de repitencia: $6,8 \%$ en la región y 7,4\% en el nivel nacional.

$\overline{4}$ La estimación de población no está incluyendo Educación Especial en apoyo direto, ni el nivel educaivo de preescolar. 
Tabla 3

Distribución de centros educativos en el Circuito 04 de la Región Educativa de Heredia por tipo de dirección

\begin{tabular}{|c|c|c|c|c|c|c|c|c|c|c|c|c|c|c|}
\hline \multirow[t]{2}{*}{ Circuito } & \multicolumn{2}{|c|}{ Unido-centes } & \multicolumn{2}{|c|}{ D1 $(*)$} & \multicolumn{2}{|c|}{ D2 } & \multicolumn{2}{|c|}{ D3 } & \multicolumn{2}{|c|}{ D4 } & \multicolumn{2}{|c|}{ D5 } & \multicolumn{2}{|c|}{ Total } \\
\hline & Abs. & $\begin{array}{l}\text { Rel. } \\
(\%)\end{array}$ & Abs. & $\begin{array}{c}\text { Rel. } \\
(\%)\end{array}$ & Abs. & $\begin{array}{l}\text { Rel. } \\
(\%)\end{array}$ & Abs. & $\begin{array}{c}\text { Rel. } \\
(\%)\end{array}$ & Abs. & $\begin{array}{c}\text { Rel. } \\
(\%)\end{array}$ & Abs. & $\begin{array}{l}\text { Rel. } \\
(\%)\end{array}$ & Abs. & $\begin{array}{l}\text { Rel. } \\
(\%)\end{array}$ \\
\hline 04 & 2 & 3,8 & 5 & 8,6 & 9 & 226,66 & 10 & 27,77 & 1 & 7,7 & 2 & 14,28 & 24 & 12,76 \\
\hline
\end{tabular}

Nota. Tomado del Departamento de Estadísticas del MEP, 2006.

(*) La letra D mayúscula hace referencia al tipo de Dirección que tiene el centro educativo, según la división administrativa establecida por el MEP, la cual define el tipo de Dirección de acuerdo con la cantidad de matrícula del centro educativo.

En la Tabla 3 puede verse que aunque la zona que abarca el circuito 04 no está alejada de las zonas urbanas, la mayor cantidad de centros educativos son dirección 2 y 3 . En la zona sólo hay dos centros educativos de dirección 5 y corresponden a los que se ubican en las cabeceras del Cantón de Barva y San Rafael, respectivamente.

Tabla 4

Circuito 04 de la Región Educativa de Heredia por tipo de institución

\begin{tabular}{|c|c|c|c|c|c|}
\hline Circuito & \multicolumn{4}{|c|}{ Tipo de institución } & No tradicional \\
\hline & PROMECUM & Horario ampliado & Excelencia & Líder & Aula abierta \\
\hline 04 & 3 & 6 & 1 & 1 & 1 \\
\hline
\end{tabular}

Nota. Tomado del Departamento de Estadísticas del MEP, 2006.

La Tabla 4 muestra los tipos de institución, según programas del MEP, que existen en el circuito 04. Llama la atención que, a pesar de que en la zona hay tres centros educativos considerados de prioridad para el Programa de Mejoramiento de Calidad de Educación y Vida en Comunidades Urbano-Marginales (PROMECUM), sólo existe un aula abierta.

Para el caso que nos ocupa, debemos mencionar que la Escuela Laboratorio funciona con horario ampliado. La solicitud se hace no por la cantidad de estudiantes, sino para poder tener tiempo de fortalecer el nuevo modelo pedagógico y dar la oportunidad, a la mayoría de sus docentes, de participar en reuniones y capacitaciones.

\section{Características generales de la Escuela Laboratorio}

Esta escuela se encuentra en Santiago de San Rafael y tiene una baja población de estudiantes (243), si tomamos como referencia su lugar de ubicación y, además, si se compara con otras escuelas con ubicaciones similares; más adelante se detallará el porqué de su baja matrícula. Se ubica sobre la carretera principal que se dirige a San Rafael y está a escasos dos kilómetros del centro de Heredia. En sus alrededores hay varias comunidades, fundamentalmente de clase media y clase 
baja. Las condiciones de infraestructura del entorno, por su ubicación, son buenas: acceso por calle principal asfaltada, luz, agua, Internet y teléfono.

La población de estudiantes, según la matrícula inicial del 2008, se distribuye de la siguiente manera:

Tabla 5

Cantidad de estudiantes según matrícula inicial (2008)

\begin{tabular}{|l|c|c|c|c|c|}
\hline & $\begin{array}{c}\text { Total de } \\
\text { estudiantes }\end{array}$ & I y II ciclos & Preescolar & $\begin{array}{c}\text { Educación } \\
\text { especial, } \\
\text { directo }\end{array}$ & $\begin{array}{c}\text { Educación } \\
\text { especial, } \\
\text { apoyo (*) }\end{array}$ \\
\hline $\begin{array}{l}\text { Dirección Regional de } \\
\text { Heredia }\end{array}$ & $\begin{array}{c}45230 \\
(100 \%)\end{array}$ & $\begin{array}{c}38189 \\
(84,4 \%)\end{array}$ & $\begin{array}{c}6672 \\
(14,8 \%)\end{array}$ & $\begin{array}{c}369 \\
(0,8 \%)\end{array}$ & $\begin{array}{c}7494 \\
(16,6 \%)\end{array}$ \\
\hline $\begin{array}{l}\text { Circuito 04 } \\
\text { Heredia }\end{array}$ & $\begin{array}{c}9226 \\
(100 \%)\end{array}$ & $\begin{array}{c}7771 \\
(84,2 \%)\end{array}$ & $\begin{array}{c}1381 \\
(15 \%)\end{array}$ & $\begin{array}{c}74 \\
(0,8 \%)\end{array}$ & $\begin{array}{c}1766 \\
(19 \%)\end{array}$ \\
\hline Escuela Laboratorio & $\begin{array}{c}243 \\
(100 \%)\end{array}$ & $\begin{array}{c}173 \\
(71,2 \%)\end{array}$ & $(24,7 \%)$ & $(4,1 \%)$ & 126 \\
& \multicolumn{3}{|r|}{} \\
\hline
\end{tabular}

Nota. Tomado del Departamento de Estadísticas del MEP 2008.

(*) Corresponde a los servicios de apoyo en Educación especial, por tanto no se suman al total de estudiantes, pues los estudiantes de I y II ciclo y preescolar pueden estar matriculados también en un servicio de apoyo.

Mediante un análisis de la tabla anterior, podemos darnos cuenta de que la distribución porcentual de la matrícula para la Escuela Laboratorio tiene un comportamiento particular, si se compara con el resto de la región educativa y del Circuito 4, ya que estos dos últimos mantienen una distribución porcentual de la matrícula igual, tanto para I y II ciclos, preescolar y educación especial, y varían solamente en un 2,4\% la relación en la cantidad de niños matriculados en los servicios de apoyo de educación especial.

Llama la atención que un porcentaje tan alto de los estudiantes de la Escuela Laboratorio estén matriculados en los servicios de apoyo de Educación especial: el 51,9\% asiste a algún servicio. La Escuela, durante el período que duró el trabajo de campo de la investigación, tenía los siguientes servicios de educación especial: apoyo fijo en problemas emocionales y de conducta, apoyo fijo en problemas de aprendizaje, aula integrada; además, contaba con el servicio de apoyo fijo en terapia del lenguaje.

Asimismo, en la Tabla 5, podemos observar que la matrícula de estudiantes en el aula integrada (servicio directo de educación especial) también es, porcentualmente, más alta que la de la población total del Circuito 04 y la de la Región Educativa en su totalidad (4\%).

La distribución porcentual de I y II ciclos y preescolar, de igual manera, varía en relación con los datos regionales.

De acuerdo con el personal del centro educativo, no es práctico seguir lo dispuesto en el Decreto 7125 sobre las Escuelas y Liceos Laboratorio, en el Artículo $\mathrm{N}^{\mathrm{o}} 6$ sobre los requerimientos para el nombramiento del personal, por el tipo de trabajo que necesitan realizar en la Escuela 
Laboratorio. Esto porque ese artículo dice que someterse a las disposiciones del Servicio Civil, hace que no siempre llegue el personal idóneo, con la disposición de trabajar en una nueva propuesta; especialmente, si muchos de los docentes laboran con los requerimientos del Ministerio de Educación (MEP) y no están dispuestos a trabajar con propuestas diferentes, ya sea por su desconocimiento, por el recelo a experimentar con propuestas nuevas o por el temor de no cumplir con las expectativas que tiene este Ministerio, en cuanto a que los estudiantes enfrenten los requisitos de evaluación impuestos por él.

El centro educativo cuenta con el siguiente personal:

\section{Personal administrativo}

- Directora

- Bibliotecaria

Personal docente

- Una docente del Ciclo materno infantil interactivo II

- Una docente del Ciclo de transición

- Una docente para cada uno de los seis grados

- Una profesora de Ética y moral

- Un profesor de Música

- Una profesora de Inglés

- Una profesora de Informática

- Un profesor de Educación física

- Un profesor de Artes plásticas

- Una profesora de Apoyo fijo en trastornos emocionales

- Una profesora de Apoyo fijo en problemas de aprendizaje

- Una profesora de Aula integrada

- Una terapista de Lenguaje

Además, cuenta con dos conserjes, dos guardas y una cocinera.

Es importante resaltar que la escuela está por encima de la media en el ámbito nacional, en cuanto a docentes que imparten cursos especiales. En este rubro, el centro educativo cuenta con seis docentes de planta, quienes tienen a su cargo diversas materias que complementan el currículo. Además, la institución cuenta con una biblioteca y servicios de educación especial, los cuales, como se mencionaba anteriormente, tienen una matrícula bastante alta según las estadísticas del MEP.

Sobre esto último, durante las visitas al centro educativo, las conversaciones con distintos funcionarios y la revisión de documentos, nunca se hizo referencia a la cantidad de estudiantes matriculados en los servicios de educación especial, a pesar de que éste fuera el tema de una de las preguntas hecha en repetidas ocasiones.

\section{Reflexiones en torno a la historia de la Escuela Laboratorio}

La Escuela Laboratorio inició sus labores en marzo de 1964, en una propiedad ubicada en el centro de Heredia. Comenzó con todos los grados de I y II ciclos de Educación Básica. Poco después, con el apoyo de la Agencia Internacional para el Desarrollo (AID), construyó un nuevo edificio contiguo a lo que fue la Escuela Normal. En 1971, abrió la sección de preescolar. 
Al principio, la escuela ofrecía charlas, demostraciones, consultas, talleres para docentes, directores, directoras y supervisores y supervisoras. La mística del personal que trabajaba en este centro educativo hacía que se dedicaran a su trabajo más allá de los horarios convencionales.

Con la apertura de la Universidad Nacional en 1973, la escuela tuvo que dejar su edificio y trasladarse a una casa, lugar en el que estuvieron hasta 1978, cuando terminaron de construir las instalaciones nuevas. El nuevo edificio se construyó con el apoyo de padres y madres de familia y algunas personas de la comunidad.

Los docentes y las docentes que estuvieron desde un inicio en el centro educativo y que dieron vida al proyecto, con los años empezaron a pensionarse o a trasladarse a trabajar a otros centros educativos. Algunas de las consideraciones que rescatan los directivos actuales sobre el cambio dado, fue la falta de incentivos para la cantidad de trabajo que realizaban.

El cuerpo docentes actual de la escuela narra que, al principio, esta recibía apoyo técnico de lo que fue la Escuela Normal Superior y que, de acuerdo con la información brindada por el personal docente de entonces: "la Escuela Laboratorio era considerada como un centro de investigación de la Escuela Normal". Ese apoyo le permitió fortalecerse como centro educativo, e iniciar los primeros pasos como una escuela laboratorio. Sin embargo, Escuela Normal pasó a manos de la Universidad Nacional y, considera que en ese momento se fue perdiendo identidad, y "se llegó en un momento a trabajar muy conductistamente y a reproducir la idea de que no pueden cambiar las metodologías de trabajo, porque son las que propone el Ministerio de Educación”. (Entrevista grupal con docentes, 4 de octubre 2006) Al respecto agregan:

Lo que sucede es que... mire... ha habido cambios administrativos, a partir de ciertas épocas de las escuelas laboratorios. Cuando yo llegué aquí, por ejemplo, había varias características de las escuelas laboratorios originales, las pizarras, los planeamientos; entonces, después, al decaer los nombramientos, va decayendo todo; también, la directora que había en esa época. Todavía, se mantenía, en alguna medida, la práctica docente. Usted sabe que la Escuela Laboratorio nació para la escuela experimental (de la Escuela Normal) que los estudiantes vinieran aquí a hacer la práctica. (Entrevista grupal a docentes, 4 de octubre 2006)

En 1977, el Poder Ejecutivo firma el Decreto $\mathbf{N}^{\circ}$ 7125, Decreto de Escuelas y Liceos Laboratorio, el cual tiene como objetivo crear las directrices de funcionamiento de un grupo de instituciones que para esa fecha están fungiendo como Escuelas Laboratorio y, a su vez, crear también la figura para los Liceos Laboratorio. Dentro de las escuelas que son consideradas y que quedarán sujetas a las disposiciones legales son: Liceo Laboratorio de San José, Escuela Laboratorio de Heredia, Unidad Pedagógica de Liberia, Escuela Laboratorio de Pérez Zeledón y la Escuela Laboratorio de San Ramón. Vale aclarar, en este punto, que la Escuela Nueva Laboratorio Emma Gamboa, funciona como un programa de la Universidad de Costa Rica, por tanto, no se contempla en el Decreto.

A pesar de ser considerada la Escuela Laboratorio de Heredia dentro del Reglamento, durante esos años, según lo indagado por esta investigación, al Decreto no se le da el seguimiento debido, pues la Escuela ya había entrado a funcionar según las exigencias del Ministerio de Educación, es decir, no había en su propuesta pedagógica proyectos orientados a la promoción y creación de estrategias innovadoras para sus educandos.

Una de las causas en la que los personeras y las personeras actuales de la Escuela ponen énfasis es en que al haberse perdido el ligamen con la Escuela Normal y no haber quedado claramente definida una relación con la Universidad Nacional, se pierde la posibilidad de convertirse en una 
institución con el recurso humano especializado, para poder dar curso a las exigencias del Decreto. "Las posibilidades de hacer desde lo que propone el MEP en sus programas habituales son pocas, si no hay un respaldo de alguna instancia académicamente reconocida" (Entrevista grupal a docentes, 4 de octubre 2006).

Otra docente agrega:

Pues bien, como la señora creía que la Universidad, exigía mucho, muchos estudiantes, mucho trabajo de parte del docente y, en realidad, qué daba...Absolutamente nada. Hubo ciertos roces y se fueron alejando en forma paulatina los estudiantes, después se dieron otros cambios y cuando en el 90 que vino otro director (a la Escuela) la Escuela perdió ese fin de escuela laboratorio, la mentalidad que él tenía era de una escuela rural, en el año 93, y no es que fuera mejor o peor, simplemente, cambió la estructura, en el año 92. También hubo un recurso de amparo aquí, presentado por los docentes, porque había problemas en lo que se refería a derechos laborales. Este recurso de amparo dice que la Escuela debe trabajar por proyectos; entonces, la visión de Escuela Laboratorio de ser un modelo experimental, donde los alumnos vienen a poner en práctica las nuevas teorías que se le enseñan en la Universidad Nacional cambió, y esa legislación supera la jurisdicción de las escuelas laboratorios, porque ustedes saben que en el momento que hay un recurso de amparo que se gana y se estimula una nueva forma de trabajo. Esta se convierte en Ley por orden de la Sala Cuarta, muy bien, entonces, ese recurso de amparo que por ahí debe de estar, dice que nosotros debemos trabajar por proyectos, entonces es así como la escuela da un giro, mucho antes que ellas empezaran a trabajar o la nueva generación de docentes, entonces, empieza a darse el proyecto de energía solar, en conjunto con la Universidad Nacional, con Juanita Coto en el año 1994, a partir de ahí dan una idea de trabajar por proyectos, nacen los proyectos de lectura, y otros muy novedosos, rehacen convenios con el grupo la nación en el año 99, han cambiado también las administraciones y, entonces, en algún momento, se dijo que era necesario tener un proyecto unificado, o integrador (2003), esa es la características de las escuelas laboratorios y todos estos proyectos están legislados en las escuelas laboratorios. (Entrevista grupal a docentes, 4 de octubre 2006)

Para el año 2004, siendo decana del Centro de Investigación y Docencia en Educación (CIDE) de la Universidad Nacional, Irma Zúñiga y Rectora Sonia Marta Mora, se plantea un convenio específico entre Universidad Nacional y el Ministerio de Educación Pública para el apoyo académico de la Escuela Laboratorio de Heredia (Centro de Investigación y Docencia en Educación (CIDE), 2010).

El 24 de febrero de 2006, el Consejo Universitario firma el convenio, con el énfasis de brindar asesoría y capacitación al personal docente de dicha institución y en setiembre del 2007 el MEP aprueba el convenio (CIDE, 2010).

Desde su aprobación participa directamente, en el apoyo al centro educativo, el Instituto de Estudios Interdisciplinarios de la Niñez y la Adolescencia (INEINA) y el programa UNA Educación de Calidad de la División de Educación Básica, ambas instancias proveen asesoría y capacitación a la Escuela Laboratorio de Heredia. 


\section{Propuesta de la Escuela Laboratorio de Heredia: un modelo pedagógico propio}

Para el año 2003, en la Escuela Laboratorio se puso en práctica un plan piloto con el fin de responder a las necesidades e intereses de sus estudiantes, mediante el uso del método de las unidades integradas. Se procuraba la implementación de diferentes métodos y técnicas pedagógicas que se adecuaran a las demandas de la sociedad actual.

El enfoque cumple a cabalidad con la misión y la visión de la Escuela Laboratorio de Heredia, abarca los contenidos programáticos del MEP y brinda a los(as) profesoras(es) de todas las materias la posibilidad de trabajar con un eje común, el cual parte del interés del estudiantado (Espinoza, Fuentes, Hernández y Jiménez, 2004).

De acuerdo con el documento que plasma el modelo pedagógico propuesto por Espinoza et al. (2004), la implementación de este promueve la formación integral del estudiante, retomando y cumpliendo con los objetivos generales para los cuales fueron creadas las escuelas laboratorio, a saber:

1. Trabajar con el objeto de capacitar a los educandos en la vida democrática, mediante su participación constante en actividades que involucren los deberes y los derechos que corresponden a todo ciudadano responsable.

2. Desarrollar investigaciones, aplicar métodos y utilizar técnicas basadas en los principios fundamentales de la psicología, la tecnología, la pedagogía y otras ciencias de la educación, con el propósito de que los educandos puedan desenvolver plenamente su personalidad y adquirir los conocimientos fundamentales en forma fácil y perdurable. Habrá en ellas centros de experimentación y documentación en tecnologías y otras disciplinas relacionadas con la educación.

3. Lograr que los estudiantes investiguen por sí mismos, reflexionen y piensen objetivamente, teniendo en cuenta que en todo problema que se confronte, existe una relación de causa y efecto.

4. Estimular el espíritu creador del educando, su capacidad para apreciar la belleza y la comprensión de los valores éticos y espirituales del grupo social al que pertenecemos. (Espinoza et al. 2004, p. 125)

En Costa Rica, las Escuelas Laboratorio fueron creadas en la década de los sesenta, como escuelas públicas para cumplir con los fines de la educación costarricense, además de servir como modelo a otros centros educativos del país, son asesoradas técnicamente por el MEP.

La misión de las Escuelas Laboratorio destaca como la de "Propiciar el desarrollo de prácticas pedagógicas innovadoras, demostrativas y experimentales, que promueven la reflexión, producción, aplicación y evaluación de nuevas alternativas técnico-pedagógicas y administrativas que se puedan divulgar y promover en todo el ámbito educativo nacional" (Espinoza et al. 2004, p. 12).

En cuanto a la visión, estas autoras consideran que:

Las Escuelas Laboratorio se constituyen en espacios educativos innovadores y centros experimentales de prácticas pedagógicas que integran el arte, la ciencia y la cultura en procesos de formación de personas críticas, participativas y reflexivas, en procura de una mejor calidad de vida individual y colectiva. Convirtiéndose así en Instituciones Modelo que favorecen el mejoramiento de la calidad de la educación del país. (Espinoza et al. 2004, p. 13) 
Las Escuelas Laboratorio tienen como filosofía trabajar por el desarrollo integral del individuo, formarlo como una persona creativa, que comprenda la realidad, que derive el significado de las cosas, elabore el conocimiento, actúe en forma adecuada frente a las diversas situaciones de la vida y construya un sistema de valores propio.

De acuerdo con Espinoza et al. (2004), la Escuela Laboratorio se identifica con una práctica de carácter activo, democrático y creativo; que motiva al niño y a la niña a adquirir nuevos conocimientos, a aprender a aprender, a aprender a hacer, a aprender a vivir en comunidad y a aprender a ser; a construir y a reconstruir su conocimiento mediante la investigación y el descubrimiento, aplicando, en interacción con otros y su medio, lo aprendido en situaciones cotidianas. Además, la Escuela Laboratorio, de acuerdo con las autoras citadas, trabaja con un mosaico de técnicas metodológicas de bases epistemológicas acordes con el paradigma socio-cultural vigente, fundamenta su accionar en la condición de que los estudiantes y las estudiantes son seres creativos, capaces de aprender a dirigir sus vidas por sí mismos. De igual forma, ve la educación como un proceso de desarrollo pleno de cada persona, que la impulsa a aprender, a pensar y a actuar libremente y con responsabilidad hacia sí misma, hacia los demás y hacia su medio.

Se concibe la tarea del personal de la escuela, de la familia y de la comunidad como la de facilitar un ambiente armonioso que propicie su integración en el mundo de significados sociales con autenticidad y con pertinencia en cuanto a sus intereses y necesidades particulares. Esto, sin olvidar los planos afectivos y motrices que integran la formación de la persona.

Se destacan, además, como funciones de la Escuela Laboratorio:

1. Formar personas críticas, analíticas, investigadoras, creativas e innovadoras.

2. Crear ambientes ricos en experiencias de aprendizaje significativo.

3. Experimentar con planteamientos teóricos, métodos y técnicas innovadoras que posibiliten el desarrollo técnico educativo.

4. Servir como institución modelo a otros centros educativos del país.

5. Difundir logros y avances de experiencias innovadoras e investigaciones que se desarrollen en su ámbito.

6. Profundizar el uso y la aplicación de nuevas tecnologías como herramienta didáctica.

7. Evaluar el impacto producido por las experiencias pedagógicas que se desarrollan en los diversos miembros de la comunidad educativa.

8. Servir a la universidad o unidad universitaria con quien tenga convenio, como laboratorio de investigaciones y prácticas pedagógicas.

9. Ofrecer asesoría a los centros de enseñanza y educadores que lo soliciten. (Espinoza et al. 2004, p. 18)

Con respecto al currículo, los docentes basan su planeamiento en los Programas del Ministerio de Educación Pública, mediante la implementación de unidades integradas fundamentadas en un enfoque constructivista "...que brinda a todos los y las docentes la posibilidad de trabajar con un eje común, tanto en las asignaturas básicas como las especiales" (Espinoza et al. 2004, p. 29). El modelo pedagógico planteado:

(...) se basa en los Programas de Estudio del Ministerio de Educación Pública y está acorde con los fines de la educación costarricense. Además, toma en cuenta los temas transversales que este propone. Otra fuente es el Lenguaje Integral y, por ende, el planeamiento también es integral. Los estudiantes seleccionan democráticamente un eje temático que es de su 
interés (aprendizaje significativo); luego, como paso siguiente, escribe qué sabe del tema, qué desea saber y cómo lo quiere investigar, a este proceso se le denomina “ preplanificación”. El docente toma todas estas inquietudes y las proyecta dentro de los distintos componentes del planeamiento, es decir: objetivos, contenidos y estrategias de mediación. (Espinoza et al. 2004, p. 34)

Entre las teorías que fundamentan la elaboración del modelo, se mencionan las de Piaget y Vygostky. En ellas, "el niño es un ser activo que se desarrolla en interacción con el ambiente" (Espinoza et al. 2004, p. 43). En este documento, las adecuaciones curriculares son consideradas como imprescindibles en el proceso de integración. Estas deben promover mayor flexibilidad de los contenidos, dar una nueva visión de los procesos de enseñanza y de aprendizaje, proporcionar soluciones a situaciones conflictivas, mostrar amplitud de criterio, fomentar la disponibilidad, creatividad y compromiso para atender las necesidades educativas especiales.

La Escuela Laboratorio trabaja con unidades integradas. En ellas se desarrollan los objetivos pertenecientes a las diferentes disciplinas (lenguaje, razonamiento matemático, entorno social, artes plásticas, informática, inglés, entre otras), así como los objetivos culturales y sociales que propician una formación integral, natural, tal y como se aprende en la cotidianeidad.

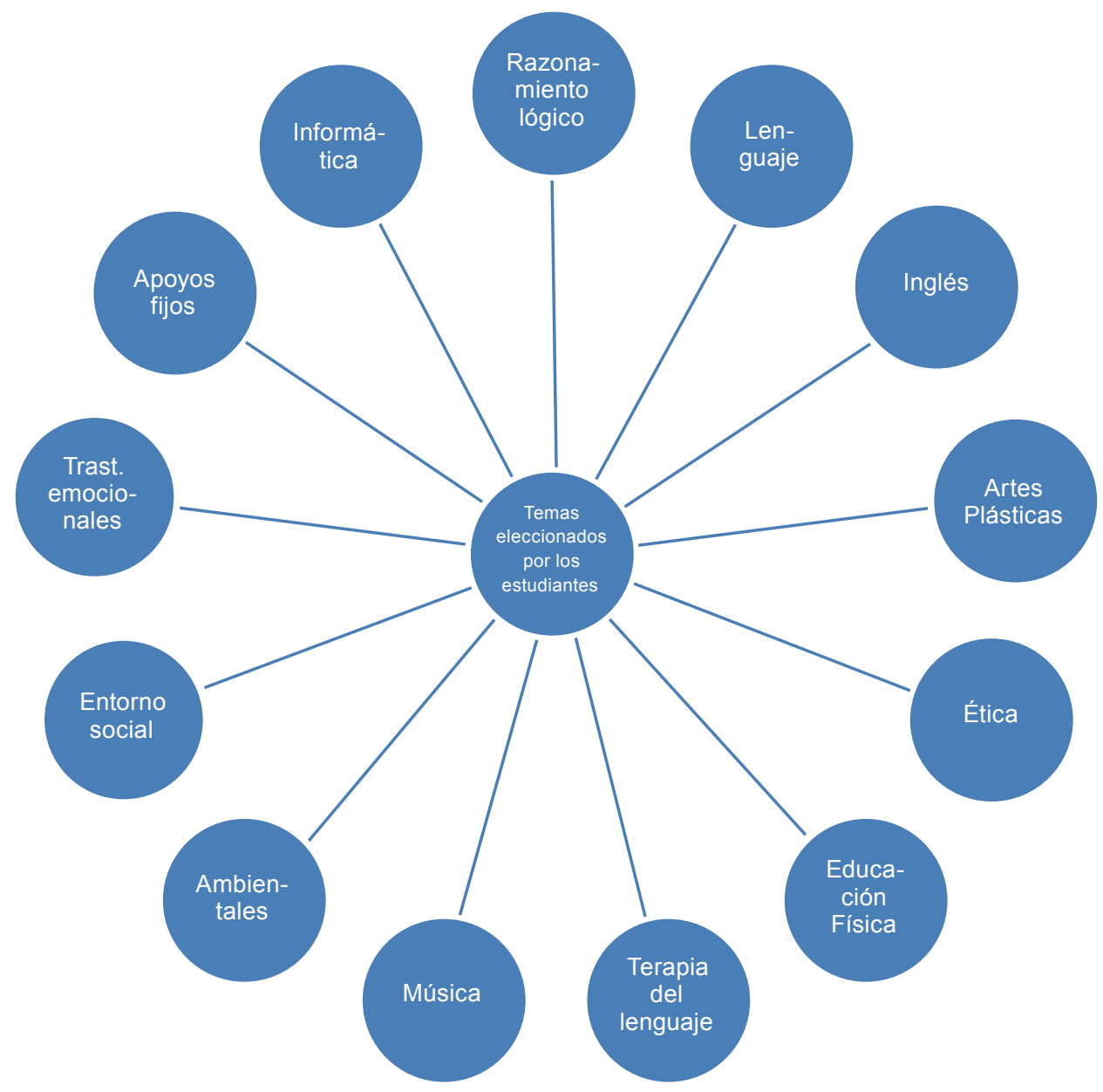

Figura 1. Esquematización del trabajo por unidades integradas que se propone en el Modelo Pedagógico de la Escuela Laboratorio de Heredia Nota. Tomado de Espinoza et al. 2004, p. 64. 
Las unidades integradas están constituidas por diferentes etapas, a saber:

1. Elección del eje temático. Los estudiantes y las estudiantes, guiados por el docente, eligen un tema de su interés, a partir del cual se elaboran las unidades integradas.

2. Pre-planificación. En relación con el tema seleccionado, los estudiantes y las estudiantes responden a las preguntas: ¿Qué sabemos de ese tema?, ¿Qué queremos saber? y ¿Cómo lo vamos a investigar? Las respuestas dadas posibilitan partir de los conocimientos de los estudiantes (conocimientos previos), orientar los objetivos hacia lo que les interesa saber y plantear situaciones de aprendizaje guiadas por las propuestas que los estudiantes brindan para abordar el tema.

3. Elaboración de las unidades integradas. Los docentes se reúnen para ayudarse unos a otros y sugerir diversas actividades para desarrollar el tema. En estos instrumentos se delimita el tiempo de estudio del tema seleccionado por los estudiantes, se plantean los objetivos generales y específicos por alcanzar y los contenidos por desarrollar, se especifican las situaciones de aprendizaje que se aplicarán, las estrategias de evaluación por utilizar y se incorporan los anexos necesarios. (Espinoza et al. 2004, p. 65)

\section{El conflicto: la puesta en práctica del nuevo modelo pedagógico}

A partir de 2002, un pequeño grupo del personal del Centro Educativo empieza a reflexionar y surge una serie de inquietudes, en torno a la verdadera identidad del centro; en ese momento se inicia la conformación de un grupo de docentes que empieza a analizar la historia de la escuela y se cuestiona sobre su verdadera identidad. Para esa misma época, y a raíz del programa Sistema Nacional de Mejoramiento de la Calidad de la Educación, el MEP se cuestiona la existencia de algunas de las escuelas laboratorio, cuyo funcionamiento había sido ratificado a partir del Decreto antes mencionado. Los cuestionamientos surgen porque se considera que estas escuelas, en realidad, no están funcionando de acuerdo con los criterios establecidos en dicho reglamento.

En 2003, se conforma el Comité para el Mejoramiento de la Calidad de la Educación, el cual sigue los lineamientos propuestos por el MEP para definir sus funciones. Además de lo establecido, este Comité se avoca a la elaboración de un Modelo pedagógico institucional.

Para realizar esta tarea, el Comité revisa todas las Actas del centro educativo, para poder identificar aquellos elementos de su historia que lo han caracterizado como una escuela laboratorio, de manera tal que diera insumo para la construcción del Modelo, y, a la vez, tener los criterios suficientes para justificar ante el MEP el funcionamiento como escuela laboratorio.

Luego de esta revisión, se hacen las primeras propuestas metodológicas en las que se enmarcarían los cambios pedagógicos en las aulas del centro educativo. Esta propuesta echa a andar en el año 2004. Todos los docentes empezaron a trabajar según los nuevos lineamientos, los cuales plantean que en el currículo de la escuela:

Los docentes basarán su planteamiento en los Programas del Ministerio de Educación Pública, mediante la implementación de Unidades integradas fundamentadas en un enfoque constructivista que brinda a todos los docentes y las docentes la posibilidad de trabajar con un eje común, tanto en las asignaturas básicas como las especiales. (Espinoza et al. 2004, p. 32) 
Ya se indicó, en páginas anteriores, que el planeamiento se basa en el Lenguaje integral.

La propuesta define que el horario de la escuela sea similar al de la Escuela Nueva Laboratorio de la UCR, ya que se considera que, con ese horario, esa escuela ha podido cumplir a cabalidad con los objetivos de ser Laboratorio, pues permite dar una mayor continuidad al proceso de enseñanza, propicia la aplicación de la teoría de las inteligencias múltiples, facilita la atención individualizada y favorece el trabajo en equipo, entre otras ventajas.

La propuesta pedagógica inicia, también, una serie de cuestionamientos y conflictos, tanto dentro del Centro Educativo como con los personeros de la Dirección Regional; estos conflictos ponen en evidencia los obstáculos que implica el cambiar la metodología tradicional que se venía utilizando.

Es así como, por ejemplo, el cambio de horario se dio mediante una negociación con la Dirección Regional para que permitiera tener un horario ampliado y que los docentes pudieran impartir lecciones hasta la 1 p.m. y tener dos horas más (hasta las 3 p.m.) para poder trabajar en capacitaciones, reuniones de equipo y talleres, entre otras actividades.

Sin embargo, este cambio de horario no abarcó a todo el personal, ya que las docentes de Educación Especial se quedaron con un horario igual al que tiene definido el Ministerio de Educación y, por tanto, no se les reconoce las horas extra que deberían trabajar con el resto del personal para las capacitaciones y discusiones. Esto hace que las docentes de Educación Especial, en cierta medida, se sientan segregadas del proceso. Esta situación se evidenció, claramente, a la hora de hacer el trabajo de campo de la investigación que sustenta este artículo, pues estas docentes desconocen, en gran medida, el Modelo pedagógico y, además, no son tomadas en cuenta para coordinar con el resto del personal del centro educativo.

Las resistencias vividas por estos cambios, al interior del centro, se pueden dividir en dos sentidos: los docentes y los padres de familia.

\section{Los docentes}

Las resistencias de estos se basa, fundamentalmente, en las implicaciones que tiene el cambio de la metodología, pues argumentan que:

a. No están capacitados para trabajar con el modelo de Unidades integradas. Aunque hay quienes opinan lo contrario, como por ejemplo:

Yo creo que el modelo, por sí solo, se enfrenta a la debilidad o la fortaleza de la persona que lo está aplicando, es algo muy interesante porque si usted lo conoce, lo estudia y lo lee, uno de los trabajos de nosotros es que debemos investigar, entonces uno supera todas esas barreras. El modelo está hecho para hacer personas creativas, autónomas, libres y para fomentar la creatividad y el autoaprendizaje; pero si la persona que los está aplicando no tiene autodisciplina no tiene una concepción libre del aprendizaje, no es por naturaleza investigadora ni creativa, problemas tiene. (Entrevista grupal con docentes, 4 de octubre 2006)

b. Los estudiantes no están preparados para poder cambiar a la nueva metodología.

c. La propuesta metodológica choca con las exigencias del MEP, tanto en el manejo de los contenidos, como en la manera de evaluar a los estudiantes. Al respecto en la entrevista realizada en el 2006 una docente de la escuela laboratorio apunta: 
Hay varias situaciones que comparto con la compañera, pero también tengo dos momentos: antes de llevar la Maestría y ahora que llevo la Maestría en la Universidad Nacional, porque antes yo participé en la elaboración, para mí en ese momento, de un modelo pedagógico, después porque, en realidad, no es un modelo pedagógico tal cual, como un modelo curricular, como lo visualizamos ahora, ya que una de las debilidades cuando se hizo el modelo pedagógico no se trabajó de manera paralela una propuesta de evaluación acorde con este modelo pedagógico, ya que había una serie de factores que intervinieron, el modelo habla de técnicas de evaluación, pero no de una propuesta de evaluación. (Entrevista grupal con docentes, 4 de octubre 2006)

d. La propuesta debe ponerse en práctica de manera paulatina.

e. Quienes han elaborado la propuesta no tienen una formación completa para poder hacerlo de manera integral.

Además, no todos los docentes comparten la propuesta, pues algunos opinan que el apoyo de la Universidad Nacional es fundamental y que, a la fecha, no se ha sabido negociar para alcanzar los objetivos propuestos:

Es importante resaltar que la Emma Gamboa (Escuela Laboratorio de la Universidad de Costa Rica) tiene muchas diferencias con la Escuela Laboratorio de Heredia; por ejemplo, ellos tienen aprobado su modelo por el Consejo (universitario); entonces, pueden hacer frente a todo este montón de críticas, de cosas negativas que se van presentando en el camino; nosotros no. Entonces, muchas veces, nos limitamos, aunque nosotros estamos conscientes de que hacemos lo mejor que podemos, que podemos cambiar, la podemos mejorar. (Entrevista grupal con docentes, 4 de octubre 2006)

Otro grupo de docentes es más optimista con el proceso, y aunque están de acuerdo con algunos de los cuestionamientos, ven como un cambio positivo en la nueva propuesta pedagógica:

Y vea, usted, que también, bueno, ese tipo de entrevista es excelente así, también porque cuando yo lo digo me resisto es porque creo más en un aprendizaje colaborativo, por cuanto le puede ayudar a una serie de limitantes, pero el lenguaje integral bien trabajado se basa en un aprendizaje colaborativo más que individualizado. (Entrevista grupal con docentes, 4 de octubre 2006)

\section{Los padres de familia}

La resistencia de la mayoría de los padres al nuevo modelo se sustenta en el temor de que sus hijos no aprendan, que no vayan a poder ser competitivos en el sistema educativo: "¿Qué pasará cuando nuestros hijos salgan de sexto grado, tendrán que repetir en el colegio?" (Tomado de reunión de padres de familia en el Centro Educativo, setiembre 2006).

Los docentes encargados de llevar adelante la propuesta conocen bien la problemática y ellos consideran que una salida para evitar mayores conflictos es cambiar la metodología de relación con los padres. Esto porque los padres consideran que cuando los niños escriben en los cuadernos, no se 
les corrige la ortografía y caligrafía, y no están de acuerdo con la evaluación que maneja el MEP, ya que se hace de una manera integrada.

De ahí surge la necesidad de proponer actividades pedagógicas que involucren más la participación de los padres, a fin de que "reconozcan el proceso de aprendizaje con sus hijos" (Entrevista grupal con docentes, 4 de octubre 2006).

A pesar de que se ha cambiado y se ha propuesto esta estrategia, no se ha logrado que el cien por ciento de los padres esté convencido de las ventajas del nuevo modelo, pues cuestionan que los maestros delegan en ellos tareas que deberían realizarse en el aula.

Otra estrategia para vincular más a los padres es aprovechar los conocimientos especializados que puedan tener. Por ejemplo, explica una docente que si un padre es dentista y en el programa se toca el tema de la salud bucal, se invita al padre para que vaya a la escuela y dé una charla, sobre ese tema, a todos los estudiantes.

Las observaciones que hace el grupo de padres que no está de acuerdo con el modelo, se pueden resumir en lo siguiente:

- $\quad$ Los contenidos que solicita el MEP no son abarcados, en su totalidad.

- Los estudiantes están rezagados, en relación con estudiantes de otros centros educativos que cursan el mismo nivel.

- $\quad$ Los maestros desconocen, a fondo, las nuevas metodologías.

- $\quad$ Los padres consideran que hay intereses de poder detrás de la propuesta. Los padres que estaban disconformes con el modelo pedagógico de la Escuela Laboratorio pidieron una reunión con la Dirección Regional para pedir aclaraciones. Los papás deseaban saber si el MEP estaba avalando ese modelo educativo.

- Los padres, también, cuestionaban si ya se había realizado una evaluación de los aspectos positivos o de aquellas debilidades del modelo. Les inquieta un poco la evaluación en relación con las pruebas de sexto que, en ese momento, se solicitaban.

- $\quad$ Los padres preguntan cómo se sitúa esta escuela con respecto al MEP.

A partir de 1996, la Escuela Laboratorio gestiona un acuerdo con la Universidad Nacional, dicho documento ya ha sido avalado por las autoridades de la Universidad y el Ministerio de Educación.

\section{A modo de conclusiones}

En la fecha en que se realizó la investigación que sustenta este artículo (2006-2007), la situación dentro de la escuela era compleja. Se podían identificar, claramente, los conflictos, en los que participan actores muy distintos con razones particulares.

Los conflictos permiten leer más allá de las nuevas condiciones que se proponen las personas que lideran el proceso a raíz de la propuesta del nuevo Modelo pedagógico. El proceso de búsqueda de su identidad como centro educativo es visto por todos como un proyecto de Escuela; sin embargo, es importante resaltar que, en el fondo, eso genera una serie de conflictos ante los cuales los distintos actores se manifiestan de manera diferente.

Es así como, a manera de síntesis, se puede decir que:

a. Quienes hacen la propuesta del nuevo modelo pedagógico y persiguen su puesta en práctica tienen una visión propia sobre lo que quieren del centro educativo, la cual no coincide con los 
imaginarios que tanto docentes como padres han construido alrededor de una visión que se considera más legítima y es la propuesta del Ministerio de Educación.

b. Se pudo encontrar como una primera situación, el cuestionamiento que hacen las autoridades del Ministerio sobre si lo que está funcionando, realmente, cumple con los fines para lo que se crea una escuela laboratorio. Los cambios, provocados por los Ministros de turno hacen que las propuestas pierdan vigencia y, por tanto, seguimiento de un gobierno a otro, pues los cuestionamientos evidencian la falta de continuidad en las propuestas o proyectos del Ministerio. Este es el caso de las Escuelas Laboratorio, las que, en este momento, no tienen un referente responsable que dé seguimiento a los procesos que realizan estos centros educativos.

c. El cuestionamiento de las autoridades regionales sobre la validez de la propuesta del nuevo modelo evidencia, también, la falta de comunicación y seguimiento de las propuestas que se dan dentro del mismo Ministerio de Educación, sobre todo, entre las oficinas centrales del Ministerio y las Direcciones Regionales.

d. La mirada de los padres y madres pone en evidencia el estereotipo viejo de aprender-memorizar como sinónimo de inteligencia, lo cual muestra la necesidad de que las propuestas innovadoras en los centros educativos deben contemplar la reeducación de los padres y madres para poder maximizar los procesos que se realizan con sus hijos e hijas.

e. Existe un juego de poder entre aquellos que consideran tienen el conocimiento sobre el modelo, aunque, en el fondo, saben que está en proceso de construcción.

f. Se presenta un divorcio entre la filosofía que sustenta el modelo y la propuesta de atención a la diversidad en el centro educativo; pues, aunque el modelo sea innovador, los números son claros: el porcentaje de servicios de educación especial que existe en el centro educativo es muy alto, y el hecho de que no participen dentro de los procesos de discusión y construcción del nuevo modelo pone en tela de duda la coherencia entre los principios epistemológicos y metodológicos sobre los que están trabajando.

La identidad escolar no es la simple descripción de la cotidianidad de un centro educativo, sino que esta se construye y reconstruye permanentemente. En el caso de la Escuela Laboratorio de Heredia, es claro que en su proyecto de centro se manifiestan distintos discursos, visiones e imaginarios, así como las distintas posiciones de los actores que están involucrados diariamente en un proyecto educativo que todos reconocen y en el cual todos participan.

\section{Referencias bibliográficas}

Centro de Investigación y Docencia en Educación [CIDE]. (2010). Archivo administrativo Registro de seguimiento a convenio con Escuela Laboratorio de Heredia. Documento sin publicar

Costa Rica. Ministerio de Planificación y Política Económica. (2005). Plan Nacional de Desarrollo 2002-2006: Informe final sobre el cumplimiento de las metas, los objetivos, las prioridades y las acciones estratégicas 2004. San José: Ministerio de Planificación y Política Económica.

Costa Rica. Poder Ejecutivo. (1977). Decreto de Escuelas y Liceos Laboratorio. D. E. \# 7125. San José, Costa Rica: Autor. 
Departamento de Estadísticas del MEP. (2008). Base de datos: Estadísticas de matrícula de I y II ciclos de educación básica, para los años 2006-2008. Documento sin publicar: Ministerio de Educación Pública

Espinoza, S., Fuentes, I., Hernández, M. y Jiménez, G. (2004). Modelo Pedagógico Institucional. Documento no publicado. 